\title{
On maximally superintegrable systems.
}

\author{
A V Tsiganov \\ St.Petersburg State University, St.Petersburg, Russia \\ e-mail: tsiganov@mph.phys.spbu.ru
}

\begin{abstract}
Locally any completely integrable system is maximally superintegrable system such as we have the necessary number of the action-angle variables. The main problem is the construction of the single-valued additional integrals of motion on the whole phase space by using these multi-valued action-angle variables. Some constructions of the additional integrals of motion for the Stäckel systems and for the integrable systems related with two different quadratic $r$-matrix algebras are discussed. Among these system there are the open Heisenberg magnet and the open Toda lattices associated with the different root systems.
\end{abstract}

\section{Introduction}

In classical mechanics a Hamiltonian system on a $2 n$-dimensional phase space $M$ is called completely integrable in Liouville's sense if it possesses $n$ functionally independent integrals of motion $H_{1}, \ldots, H_{n}$ in the involution:

$$
\frac{d H_{i}}{d t}=\left\{H, H_{i}\right\}=0, \quad\left\{H_{i}, H_{j}\right\}=0, \quad i, j=1, \ldots, n,
$$

where $H=H_{1}$ is the Hamilton function and $\{.,$.$\} is the Poisson bracket on M$.

A superintegrable system is a system that is integrable in the Liouville sense and that, in addition to this, possesses more functionally independent integrals of motion than degrees of freedom. If the number of independent integrals takes the value $2 n-1$, then the system is called maximally superintegrable [14. There are three classic and well-known examples of such systems, namely, the free particle that can be considered as trivial, the Kepler problem, and the harmonic oscillator with rational frequencies. In these cases all the orbits become closed for the case of bounded motions.

The Liouville classical theorem on completely integrable Hamiltonian systems implies that almost all points of the manifold $M$ are covered by a system of open toroidal domains with the action-angle coordinates $I=\left(I_{1}, \ldots, I_{k}\right)$ and $\omega=\left(\omega_{1}, \ldots, \omega_{n}\right)$ [7]:

$$
\left\{I_{j}, I_{k}\right\}=\left\{\omega_{i}, \omega_{k}\right\}=0, \quad\left\{I_{j}, \omega_{k}\right\}=\delta_{i j} .
$$

The independent integrals of motion $H_{1}, \ldots, H_{n}$ are functions upon the independent action variables $I_{1}, \ldots, I_{n}$ and the corresponding Jacobian does not equal to zero

$$
\operatorname{det} \mathbf{J} \neq 0, \quad \text { where } \quad \mathbf{J}_{i j}=\frac{\partial H_{i}\left(I_{1}, \ldots, I_{n}\right)}{\partial I_{j}} .
$$

Let us introduce $n$ functions

$$
\phi_{j}=\sum_{k}\left(\mathbf{J}^{-1}\right)_{k j} \omega_{k}
$$

such that

$$
\left\{H_{i}, \phi_{j}\right\}=\sum_{k=1}^{n} \mathbf{J}_{i k}\left(\mathbf{J}^{-1}\right)_{k j}=\delta_{i j} .
$$


The $(n-1)$ functions $\phi_{2}, \ldots, \phi_{n}$ are integrals of motion

$$
\frac{d \phi_{j}}{d t}=\left\{H_{1}, \phi_{j}\right\}=0, \quad j=2, \ldots, n,
$$

which are functionally independent on $n$ functions $H_{1}(I), \ldots, H_{n}(I)$. So, in classical mechanics any completely integrable system is superintegrable system in a neighborhood of any regular point of $M$. It means that the Hamiltonian $H=H_{1}$ has $2(n-1)$ integrals of motion $H_{2}, \ldots, H_{n}$ and $\phi_{2}, \ldots, \phi_{n}$ on any open toroidal domain.

Sometimes the action-angle variables are global variables on the whole phase space $M$ and, therefore, we have superintegrable systems on $M$. For instance, the global action-angle variables for the open and periodic Toda lattices are discussed in [5].

In generic case the action variables $\omega_{k}$ are multi-valued functions on the whole phase space $M$. In this case if we have some algebraic integral of motion $K$, it has to be some function on the action-angle variables. In this paper we discuss some possibilities to get polynomial integrals of motion from the multi-valued angle variables.

\section{The Stäckel systems.}

The system associated with the name of Stäckel [13, 15, 16] is a holonomic system on the phase space $\mathbb{R}^{2 n}$, with the canonical variables $q=\left(q_{1}, \ldots, q_{n}\right)$ and $p=\left(p_{1}, \ldots, p_{n}\right)$ :

$$
\Omega=\sum_{j=1}^{n} d p_{j} \wedge d q_{j}, \quad\left\{p_{j}, q_{k}\right\}=\delta_{j k} .
$$

The nondegenerate $n \times n$ Stäckel matrix $S$, whose $j$ column depend on coordinate $q_{j}$ only, defines $n$ functionally independent integrals of motion

$$
H_{k}=\sum_{j=1}^{n}\left(S^{-1}\right)_{j k}\left(p_{j}^{2}+U_{j}\left(q_{j}\right)\right) .
$$

From this definition one immediately gets the separated relations

$$
p_{j}^{2}=\sum_{k=1}^{n} H_{k} S_{k j}\left(q_{j}\right)-U_{j}\left(q_{j}\right) \text {. }
$$

It allows reducing the solution of the equations of motion to a problem in algebraic geometry [2, 4, 15]. We can regard each separated equation (2.3) as equation defining the hyperelliptic curve

$$
\mathcal{C}_{j}: \quad \mu_{j}^{2}=P_{j}\left(\lambda_{j}\right),
$$

where $\mu_{j}$ and $\lambda_{j}$ are functions on $p_{j}$ and $q_{j}$ only and $P_{j}\left(\lambda_{j}\right)$ are polynomials.

The functionally independent action variables $I_{k}=H_{k}(2.2)$ have the canonical Poisson brackets (1.1) with the following angle variables

$$
\omega_{i}=\sum_{j=1}^{n} \int_{A_{j}} \frac{S_{i j}\left(\lambda_{j}\right)}{\sqrt{P_{j}\left(\lambda_{j}\right)}} \mathrm{d} \lambda_{j},
$$

which consist of integrals of first kind Abelian differentials on the hyperelliptic curves $C_{j}$ (2.4) [2, 4, 15, 16. For the coinciding curves we have to use different $A$-cycles on the Riemann surfaces in the sums (2.5).

In generic case the action variables (2.5) are the sum of the multi-valued functions $\vartheta_{i j}$. However, if we are able to apply some addition theorem to the calculation of $\omega_{i}$ (2.5)

$$
\omega_{i}=\sum_{j=1}^{n} \vartheta_{i j}\left(p_{j}, q_{j}\right)=\Theta_{i}\left(K_{i}\right),
$$


where $\Theta_{i}$ is a multi-valued function on the algebraic argument $K_{i}(p, q)$ then one gets algebraic integrals of motion $K_{i}(p, q)$ such that

$$
\left\{H_{1}, \omega_{i}\right\}=\left\{H_{1}, \Theta_{i}\left(K_{i}\right)\right\}=\Theta_{i}^{\prime} \cdot\left\{H_{1}, K_{i}\right\}=0 .
$$

So, the addition theorems (2.6) could help us to classify algebraically superintegrable systems and vice versa.

Remark 1 We can express hyperelliptic integrals $\left.\vartheta_{i j}(2.5] \mid 2.6\right)$ via the elementary functions for the hyperelliptic curves $\mathcal{C}_{j}$ with genus $=0$ only [2, 4. Namely, if

$$
\operatorname{deg} P_{j}(\lambda)>2,
$$

then variable $\omega_{j}$ (2.5) are elliptic functions on $p$ and $q$.

Remark 2 According to [6] construction of the separated variables $(q, p)$ for the $L$-subset of the Stäckel systems is a pure computer problem. The software [6] may be easily improved in order to calculate additional integrals of motion $\omega_{k}(2.5)$ too.

\section{Example 1 The (b) Drach system}

Let us consider the Stäckel system defined by

$$
S=\left(\begin{array}{cc}
q_{1}^{2} & q_{2}^{2} \\
1 & 1
\end{array}\right), \quad U_{1,2}= \pm 2 \alpha-\frac{\beta \mp 2 \gamma}{q_{1,2}^{2}}
$$

such that the separated relations (2.3) look like

$$
p_{1,2}^{2}=H_{1} q_{1,2}^{2}+H_{2} \pm 2 \alpha-\frac{\beta \mp 2 \gamma}{q_{1,2}^{2}} .
$$

In variables $\mu_{j}=q_{j} p_{j}$ and $\lambda_{j}=q_{j}^{2}$ equations (2.4) became the canonical equations defining two Riemann surfaces:

$$
\mathcal{C}_{1,2}: \quad \mu^{2}=P_{1,2}(\lambda)=H_{1} \lambda^{2}+H_{2} \lambda \pm 2 \alpha \lambda-\beta \pm 2 \gamma .
$$

The action variables $I_{1,2}=H_{1,2}$ have the canonical brackets (1.1) with the following angle variables (2.5):

$$
\begin{aligned}
& \omega_{1}=\frac{1}{4}\left(\int^{q_{1}^{2}} \frac{\lambda}{\sqrt{P_{1}(\lambda)}} \mathrm{d} \lambda+\int^{q_{2}^{2}} \frac{\lambda}{\sqrt{P_{2}(\lambda)}} \mathrm{d} \lambda\right), \\
& \omega_{2}=\frac{1}{4}\left(\int^{q_{1}^{2}} \frac{1}{\sqrt{P_{1}(\lambda)}} \mathrm{d} \lambda+\int^{q_{2}^{2}} \frac{1}{\sqrt{P_{2}(\lambda)}} \mathrm{d} \lambda\right) .
\end{aligned}
$$

So, the Hamiltonian system with hamiltonian $H_{1}$ has two independent integrals of motion $\mathrm{H}_{2}$ (2.2) and

$$
\omega_{2}=\frac{1}{4 \sqrt{H_{1}}}\left[\ln \left(p_{1} q_{1}+\frac{P_{1}^{\prime}}{2 \sqrt{H_{1}}}\right)+\ln \left(p_{2} q_{2}+\frac{P_{2}^{\prime}}{2 \sqrt{H_{1}}}\right)\right],
$$

where

$$
P_{1,2}^{\prime}=\left.\frac{d P_{1,2}(\lambda)}{d \lambda}\right|_{\lambda=q_{j}^{2}}=2 H_{1} q_{1,2}^{2}+H_{2} \pm 2 \alpha .
$$

Using addition theorem

$$
e^{x} \mathrm{e}^{y}=\mathrm{e}^{x+y} \quad \text { or } \quad \ln \left(x_{1}\right)+\ln \left(x_{2}\right)=\ln \left(x_{1} x_{2}\right)
$$

one gets

$$
\omega_{2}=\frac{1}{4 \sqrt{H_{1}}} \ln \left[\left(p_{1} q_{1}+\frac{P_{1}^{\prime}}{2 \sqrt{H_{1}}}\right)\left(p_{2} q_{2}+\frac{P_{2}^{\prime}}{2 \sqrt{H_{1}}}\right)\right] .
$$


So we can introduce algebraic integral of motion

$$
\begin{aligned}
\Phi_{1}(I, \omega) & =\mathrm{e}^{4 \sqrt{H_{1}} \omega_{2}}=\left(p_{1} q_{1}+\frac{P_{1}^{\prime}}{2 \sqrt{H_{1}}}\right)\left(p_{2} q_{2}+\frac{P_{2}^{\prime}}{2 \sqrt{H_{1}}}\right)= \\
& =\frac{4 p_{1} p_{2} q_{1} q_{2} H_{1}+P_{1}^{\prime} P_{2}^{\prime}}{4 H_{1}}+\frac{p_{1} q_{1} P_{2}^{\prime}+p_{2} q_{2} P_{1}^{\prime}}{2 \sqrt{H_{1}}}= \\
& =\frac{K_{4}}{4 H_{1}}+\frac{K_{3}}{2 \sqrt{H_{1}}},
\end{aligned}
$$

where $K_{4}$ and $K_{3}$ are polynomials fourth and third order in momenta

$$
K_{3}=2\left(p_{1} q_{1} P_{2}^{\prime}+p_{2} q_{2} P_{1}^{\prime}\right), \quad K_{4}=P_{1}^{\prime} P_{2}^{\prime}+4 p_{1} q_{1} p_{2} q_{2} H_{1},
$$

such that

$$
\left\{H_{1}, K_{3}\right\}=\left\{H_{1}, K_{4}\right\}=0 \quad \text { and } \quad\left\{H_{2}, K_{3}\right\}=K_{4} .
$$

After the following change of variables

$$
x=\frac{\left(q_{1}-q_{2}\right)^{2}}{4}, \quad p_{x}=\frac{p_{1}-p_{2}}{q_{1}-q_{2}}, \quad y=\frac{\left(q_{1}+q_{2}\right)^{2}}{4}, \quad p_{y}=\frac{p_{1}+p_{2}}{q_{1}+q_{2}},
$$

the Hamiltonian

$$
H_{1}=p_{x} p_{y}+\frac{\alpha}{\sqrt{x y}}+\frac{\beta}{(x-y)^{2}}+\frac{\gamma(x+y)}{\sqrt{x y}(x-y)^{2}}
$$

coincides with the Hamiltonian for the one of the Drach systems (case (b) in [17]), whereas cubic polynomial $K_{2}$ is the Drach integral of motion [17.

\section{Example 2 The (1) Drach system.}

Let us consider another Drach system (case (1) in [17]) with the Hamiltonian

$$
H_{1}=p_{x} p_{y}+\alpha\left(y-\frac{\rho x}{3}\right)+\beta x^{-1 / 2}+\gamma x^{-1 / 2}(y-\rho x)
$$

Without lost of generality we can put $\rho=-3$. Substituting this Hamiltonian into the computer programm from [6] one gets the separated variables

$$
x=\frac{\left(q_{1}-q_{2}\right)^{2}}{2}, \quad y=\frac{\left(q_{1}+q_{2}\right)^{2}}{2}
$$

and the corresponding separated relations

$$
p_{j}^{2}=P_{j}\left(q_{j}\right)=-4 \alpha q_{j}^{4} \mp 8 \sqrt{2} \gamma q_{j}^{3}+4 H_{1} q_{1}^{2} \mp 4 \sqrt{2} \beta q_{j}+H_{2}, \quad j=1,2,
$$

which give rise a pair of hyperelliptic curves at $\mu_{j}=p_{j}$ and $\lambda_{j}=q_{j}$. Using the Stäckel matrix

$$
S=\left(\begin{array}{cc}
4 q_{1}^{2} & 4 q_{2}^{2} \\
1 & 1
\end{array}\right)
$$

we can get the angle variable

$$
\omega_{2}=\frac{1}{2} \int^{q_{1}} \frac{\mathrm{d} \lambda}{\sqrt{P_{1}(\lambda)}}+\frac{1}{2} \int^{q_{2}} \frac{\mathrm{d} \lambda}{\sqrt{P_{2}(\lambda)}}
$$

which is a sum of the incomplete elliptic integrals of the first kind. We do not know how to get algebraic integral of motion starting with this variable $\omega_{2}$.

However, we know that there is additional cubic integral of motion

$$
K_{3}=2\left(\widetilde{P}_{1}^{\prime} p_{2}+\widetilde{P}_{2}^{\prime} p_{1}\right)
$$


which looks like as the Drach integral (2.8), but in this case functions

$$
\widetilde{P}_{j}^{\prime}=\left.\left(\lambda_{1}+\lambda_{2}\right)^{2} \frac{\partial}{\partial q_{j}} \frac{P_{j}\left(\lambda_{j}\right)}{\left(\lambda_{1}+\lambda_{2}\right)^{4}}\right|_{\lambda_{1,2}=q_{1,2}}
$$

have not simple algebro-geometric explanation. Nevertheless, this polynomial integral $K_{3}$ has to be a function on polynomials $H_{1,2}$ and elliptic function $\omega_{2}$. Since, the sum of elliptic integrals

$$
\omega_{2}=F\left(H_{1}, H_{2}, K_{3}\right)
$$

has to be some function on polynomials $H_{1,2}$ and $K_{3}$.

It will be interesting to get this function explicitly and to understand why it exists.

\section{Example 3 The Henon-Heiles system}

Let us consider the Stäckel system defined by

$$
S=\left(\begin{array}{cc}
1 & 1 \\
1 & -1
\end{array}\right), \quad U_{1,2}=a q_{1,2}^{3},
$$

such that

$$
p_{1,2}^{2}=H_{1} \pm H_{2}+a q_{1,2}^{3} .
$$

The action variables

$$
I_{1}=H_{1}=\frac{p_{1}^{2}+p_{2}^{2}}{2}-\frac{a\left(q_{1}^{3}+q_{2}^{3}\right)}{2}, \quad I_{2}=H_{2}=\frac{p_{1}^{2}-p_{2}^{2}}{2}-\frac{a\left(q_{1}^{3}-q_{2}^{3}\right)}{2}
$$

have the canonical brackets (1.1) with the angle variables (2.5):

$$
w_{1,2}=\frac{1}{2}\left(\int^{q_{1}} \frac{\mathrm{d} \lambda}{\sqrt{a \lambda^{3}+H_{1}+H_{2}}} \pm \int^{q_{2}} \frac{\mathrm{d} \lambda}{\sqrt{a \lambda^{3}+H_{1}-H_{2}}}\right) .
$$

So, the Hamiltonian system with the hamiltonian $H_{1}$ has two independent integrals of motion $\mathrm{H}_{2}(2.10)$ and

$$
\omega_{2}=\operatorname{const}\left(\frac{F\left(\arcsin \left(z_{1}\right), \kappa\right)}{\left(H_{1}+H_{2}\right)^{1 / 6}}-\frac{F\left(\arcsin \left(z_{1}\right), \kappa\right)}{\left(H_{1}-H_{2}\right)^{1 / 6}}\right),
$$

where $F(z, \kappa)$ is the incomplete elliptic integral of the first kind and

$$
z_{1,2}=\frac{(-1)^{11 / 12}}{3^{1 / 4}} \sqrt{1+\frac{a^{1 / 3} q_{1,2}}{\left(H_{1} \pm H_{2}\right)^{1 / 3}}}, \quad \kappa=(-1)^{1 / 3} .
$$

After the following change of variables

$$
q_{1,2}=\frac{x \pm y}{2}, \quad p_{1,2}=p_{x} \pm p_{y}
$$

the Hamilton function $H_{1}(2.10)$

$$
H_{1}=p_{x}^{2}+p_{y}^{2}-\frac{a x\left(x^{2}+3 y^{2}\right)}{8}
$$

coincides with one of the Henon-Heiles hamiltonians.

Of course, integral of motion $\omega_{2}$ (2.11) remains an elliptic function in any coordinates and we can not get single-valued integral of motion on the whole phase space. 


\section{The Sklyanin algebra}

In this section we study a class of finite-dimensional Liouville integrable systems described by the representations of the quadratic $r$-matrix Poisson algebra, or the Sklyanin algebra:

$$
\{\stackrel{1}{T}(\lambda), \stackrel{2}{T}(\mu)\}=[r(\lambda-\mu), \stackrel{1}{T}(\lambda) \stackrel{2}{T}(\mu)]
$$

Here $\stackrel{1}{T}(\lambda)=T(\lambda) \otimes \mathrm{Id}, \stackrel{2}{T}(\mu)=\mathrm{Id} \otimes T(\mu)$ and $r(\lambda-\mu)$ is a classical $r$-matrix [8]-[10]. In the simplest case of the $4 \times 4$ rational $r$-matrix

$$
r(\lambda-\mu)=\frac{\eta}{\lambda-\mu} \Pi, \quad \Pi=\left(\begin{array}{llll}
1 & 0 & 0 & 0 \\
0 & 0 & 1 & 0 \\
0 & 1 & 0 & 0 \\
0 & 0 & 0 & 1
\end{array}\right), \quad \eta \in \mathbb{C},
$$

matrix $T(\lambda)$ depends polynomially on the parameter $\lambda$

$$
\begin{aligned}
T(\lambda) & =\left(\begin{array}{ll}
A(\lambda) & B(\lambda) \\
C(\lambda) & D(\lambda)
\end{array}\right) \\
& =\left(\begin{array}{ll}
\alpha \lambda^{n}+A_{1} \lambda^{n-1}+\ldots+A_{n} & \beta \lambda^{n}+B_{1} \lambda^{n-1}+\ldots+B_{n} \\
\gamma \lambda^{n}+C_{1} \lambda^{n-1}+\ldots+C_{n} & \delta \lambda^{n}+D_{1} \lambda^{n-1}+\ldots+D_{n}
\end{array}\right) .
\end{aligned}
$$

The leading coefficients $\alpha, \beta, \gamma, \delta$ and $2 n$ coefficients of the $\operatorname{det} T(\lambda)$

$$
d(\lambda)=\operatorname{det} T(\lambda)=(\alpha \delta-\beta \gamma) \lambda^{2 n}+Q_{1} \lambda^{2 n-1}+\cdots+Q_{2 n} .
$$

are Casimirs of the bracket (3.1). Therefore, we have a $4 n$-dimensional space of the coefficients $A_{i}, B_{i}, C_{i}$ and $D_{i}$ with $2 n$ Casimir operators $Q_{i}$, leaving us with $n$ degrees of freedom.

\subsection{Open lattices}

For so-called open lattices independent Poisson involutive integrals of motion are given by the coefficients of the entry $A(\lambda)$ :

$$
A(\lambda)=\alpha \lambda^{n}+H_{1} \lambda^{n-1}+\cdots H_{n}, \quad\left\{H_{k}, H_{m}\right\}=0 .
$$

In the special action-angle representation [19], one has $n$ pairs of the action-angle variables:

$$
A\left(I_{m}\right)=0, \quad \omega_{m}=\eta^{-1} \ln B\left(I_{m}\right), \quad m=1, \ldots, n .
$$

having the standard Poisson brackets (1.1).

Since the action variables $I_{k}$ are zeroes of the polynomial

$$
A(\lambda)=\alpha \lambda^{n}+H_{1} \lambda^{n-1}+\cdots H_{n}=\alpha \prod_{m=1}^{n}\left(\lambda-I_{m}\right),
$$

initial integrals of motion $H_{m}$ are elementary symmetric function on $I_{m}$

$$
H_{1}=-\sum_{m=1}^{n} I_{m}, \quad H_{2}=\sum_{k \neq m}^{n} I_{k} I_{m}, \quad \ldots, \quad H_{n}=(-1)^{n} \prod_{m=1}^{n} I_{m}
$$

and the matrix $\mathbf{J}(1.2)$ is equal to

$$
\mathbf{J}=\left(\begin{array}{ccc}
-1 & \cdots & -1 \\
\sum_{m \neq 1} I_{m} & \cdots & \sum_{m \neq n} I_{m} \\
\vdots & \ddots & \vdots \\
(-1)^{n} \prod_{m \neq 1} I_{m} & \cdots & (-1)^{n} \prod_{m \neq n} I_{m}
\end{array}\right) .
$$


In this case all the functions $\phi_{k}$ (1.3) are functionally independent and one gets that open lattices related with the Sklyanin algebra are maximally superintegrable systems.

We have to underline that functions $\omega_{k}$ (3.6) may be found without integration, i.e. using pure algebraic constructions. As for the (b) Drach system one gets

$$
\left\{I_{i}, w_{j}\right\}=\left\{I_{i}, \ln B\left(I_{j}\right)\right\}=0, \quad \Rightarrow \quad\left\{I_{i}, B\left(I_{j}\right)\right\}=0, \quad i \neq j .
$$

So, for any Hamiltonian $I_{i}$ we have additional integrals of motion $B\left(I_{j}\right)$, which are polynomials on momenta and the remaining action variables $I_{j}$.

\subsection{Periodic lattices}

For so-called periodic lattices integrals of motion are given by the coefficients of the trace of $T(\lambda)$ :

$$
\tau(\lambda)=\operatorname{tr} T(\lambda)=(\alpha+\delta) \lambda^{n}+H_{1} \lambda^{n-1}+\cdots H_{n}, \quad\left\{H_{i}, H_{j}\right\}=0 .
$$

According to [8]-[10], in this case the separated coordinates $u_{i}, v_{i}$ defined by

$$
B\left(u_{i}\right)=0, \quad v_{i}=A\left(u_{i}\right), \quad i=1, \ldots, n,
$$

satisfy to the following separated relations

$$
v_{i}+\operatorname{det} T\left(u_{i}\right) v_{i}^{-1}=A\left(u_{i}\right)+D\left(u_{i}\right)=\tau\left(u_{i}\right), \quad i=1, \ldots, n .
$$

The corresponding symplectic form in $(u, v)$-variables is written as

$$
\Omega=\eta \sum_{j=1}^{n} \mathrm{~d} \log \left(v_{j}\right) \wedge \mathrm{d} u_{j}
$$

Let us take the action coordinates $I_{m}=H_{m}$ (3.7) instead of $v$-variables [10, 11]. From (3.8) one gets

$$
v_{j}=A\left(u_{j}\right)=\frac{1}{2}\left(\tau\left(u_{j}\right)+\sqrt{\tau\left(u_{j}\right)-4 d\left(u_{j}\right)}\right)
$$

and one easily find expression for the symplectic form in $(I, u)$-variables

$$
\Omega=\eta \sum_{j=1}^{n} \sum_{k=1}^{n} \frac{u_{j}^{n-k}}{\sqrt{P\left(u_{j}\right)}} \mathrm{d} I_{k} \wedge \mathrm{d} u_{j},
$$

where $P(\lambda)=\tau^{2}(\lambda)-4 d(\lambda)$. The equations of motion

$$
\left\{\tau(\lambda), u_{j}\right\}=\eta \sqrt{P\left(u_{j}\right)} \prod_{k \neq j} \frac{\lambda-u_{k}}{u_{j}-u_{k}}
$$

are linearized by the Abel transformation [2, 4, and the corresponding angle variables look like

$$
\omega_{j}=\eta \sum_{k=1}^{n} \int^{u_{k}} \frac{\lambda^{n-j}}{\sqrt{P(\lambda)}} \mathrm{d} \lambda, \quad \operatorname{deg} P(\lambda)=2 n .
$$

In this case matrix $\mathbf{J}(1.2)$ is identity matrix and integrals of motion $\phi_{k}=\omega_{k}(3.10)$ are elliptic functions and we could not get single-valued additional integral of motion on the whole phase space.

Recall, that quadratic $r$-matrix algebra (3.1) is related with a set of integrable systems that includes $X X X$ Heisenberg magnet, the Toda lattices, the discrete self-trapping model and the Goryachev-Chaplygin gyrostat [19]. 


\section{Example 4 The Heisenberg magnet}

Our main model turns out to be an $n$-site Heisenberg magnet, which is an integrable lattice of $n \mathrm{sl}(2)$ spins with nearest neighbor interaction. In the lattice representation the matrix $T(\lambda)$ (4.2) acquires the following form:

$$
T(\lambda)=L_{1}\left(\lambda-c_{1}\right) L_{2}\left(\lambda-c_{2}\right) \cdots L_{n}\left(\lambda-c_{n}\right),
$$

with

$$
L_{i}(\lambda)=\left(\begin{array}{cc}
u-s_{i}^{3} & s_{i}^{+} \\
s_{i}^{-} & u+s_{i}^{3}
\end{array}\right), \quad i=1, \ldots, n .
$$

Here the local variables $s_{i}^{\alpha}, i=1, \ldots, n$, are generators of $n$ copies of the sl(2) Poisson algebra:

$$
\left\{s_{j}^{3}, s_{j}^{ \pm}\right\}= \pm s_{j}^{ \pm}, \quad\left\{s_{j}^{+}, s_{j}^{-}\right\}=2 s_{j}^{3} .
$$

and $c_{m}$ are arbitrary numbers. If $n=2$ one gets

$$
A(\lambda)=\lambda^{2}+H_{1} \lambda+H_{2}=\left(\lambda-I_{1}\right)\left(\lambda-I_{2}\right),
$$

where

$$
H_{1}=-\left(s_{1}^{3}+s_{2}^{3}+c_{1}+c_{2}\right), \quad H_{2}=s_{1}^{3} s_{2}^{3}+s_{1}^{+} s_{2}^{-}+c_{1} s_{2}^{3}+c_{2} s_{1}^{3}+c_{1} c_{2},
$$

and

$$
I_{1,2}=-\frac{1}{2}\left(H_{1} \pm \sqrt{H_{1}^{2}-4 H_{2}}\right)
$$

The second polynomial

$$
B(\lambda)=\left(s_{1}^{+}+s_{2}^{+}\right) \lambda-s_{1}^{2} s_{2}^{+}+s_{1}^{+} s_{2}^{3}-c_{1} s_{2}^{+}-c_{2} s_{1}^{+}
$$

defines the angle variables

$$
\omega_{1,2}=\ln B\left(I_{1,2}\right)
$$

The functions $\phi_{1,2}(1.3)$

$$
\phi_{1}=-\frac{I_{1} \omega_{1}-I_{2} \omega_{2}}{I_{1}-I_{2}}, \quad \phi_{2}=-\frac{\omega_{1}-\omega_{2}}{I_{1}-I_{2}} .
$$

obey to equations $\left\{H_{i}, \phi_{j}\right\}=\delta_{i j}$. So, the Hamiltonian system with the hamiltonian $H_{2}$ has two independent integrals of motion $H_{1}$ and

$$
\Phi=-\left(I_{1}-I_{2}\right) \phi_{1}=I_{1} \omega_{1}-I_{2} \omega_{2}=I_{1} \ln B\left(I_{1}\right)-I_{2} \ln B\left(I_{2}\right) .
$$

The similar additional integral for the periodic lattice is some combination of elliptic functions even in the simplest case $n=2$.

\section{Example 5 The Toda lattice}

The Toda lattices appear as a specialization of our basic model when the parameters are fixed as follows:

$$
\beta=\gamma=\delta=0 \quad \text { and } \quad \operatorname{det} T(\lambda)=1 .
$$

We also put $\alpha=1$ and $\eta=-1$. In the lattice representation, the monodromy matrix $T$ (4.2) acquires the form

$$
T(\lambda)=L_{1}(\lambda) \cdots L_{n-1}(\lambda) L_{n}(\lambda), \quad L_{i}=\left(\begin{array}{cc}
\lambda-p_{i} & -\mathrm{e}^{q_{i}} \\
\mathrm{e}^{-q_{i}} & 0
\end{array}\right) .
$$

Here $p_{i}, q_{i}$ are Darboux variables $\left\{q_{i}, p_{j}\right\}=\delta_{i j}$. If $n=2$ one gets

$$
A(\lambda)=\lambda^{2}-\left(p_{1}+p_{2}\right) \lambda+p_{1} p_{2}-\mathrm{e}^{q_{1}-q_{2}}=\left(\lambda-I_{1}\right)\left(\lambda-I_{2}\right),
$$


and

$$
B=-e^{q_{2}}\left(\lambda-p_{1}\right)
$$

Let us consider natural Hamiltonian systems with integrals of motion

$$
\tilde{H}_{1}=-H_{1}=p_{1}+p_{2}, \quad \tilde{H}_{2}=\frac{p_{1}^{2}+p_{2}^{2}}{2}+\mathrm{e}^{q_{1}-q_{2}}=\frac{H_{1}^{2}}{2}-H_{2} .
$$

For these integrals we have

$$
\tilde{\mathbf{J}}=\left(\begin{array}{cc}
1 & 1 \\
I_{1} & I_{2}
\end{array}\right), \quad \text { and } \quad \tilde{\phi}_{1}=-\frac{I_{1} \omega_{2}-I_{2} \omega_{1}}{I_{1}-I_{2}}, \quad \tilde{\phi}_{2}=-\frac{\omega_{1}-\omega_{2}}{I_{1}-I_{2}}
$$

So, the Hamiltonian system with the hamiltonian $\tilde{H}_{2}$ has two independent integrals of motion $\tilde{H}_{1}$ and

$$
\Phi_{1}=-\left(I_{1}-I_{2}\right) \tilde{\phi}_{1}=I_{1} \omega_{2}-I_{2} \omega_{1}=-\left(I_{1} \ln B\left(I_{2}\right)-I_{2} \ln B\left(I_{1}\right)\right) .
$$

Namely this "generalized angular momentum" has been found in [1, 3]. In contrast with the Drach system we can not directly apply additional theorem in this case.

At the periodic case one gets

$$
I_{1}=-\left(p_{1}+p_{2}\right), \quad I_{2}=p_{1} p_{2}-\mathrm{e}^{q_{1}-q_{2}}-\mathrm{e}^{q_{2}-q_{1}},
$$

and the Hamiltonian system with the hamiltonian $H=I_{2}$ has two independent integrals $I_{1}$ and

$$
\phi_{1}=-\frac{q_{1}+q_{2}}{2}+\frac{I_{1} w_{2}}{2}, \quad \text { where } \quad \omega_{2}=\int^{p_{1}} \frac{\mathrm{d} \lambda}{\sqrt{\left(\lambda^{2}+I_{1} \lambda+I_{2}\right)^{2}-4}} .
$$

As for Henon-Heiles system this additional integral of motion may be rewritten as a combination of elliptic functions on $p_{1}$ and $I_{1,2}$.

The global action-angle variables for the periodic Toda lattices are discussed in [5]. Nevertheless we suppose that periodic Toda lattices are locally maximally superintegrable systems only.

\section{Reflection equation algebra}

In this section we study a class of finite-dimensional Liouville integrable systems described by the representations of the reflection equation algebra:

$$
\begin{aligned}
\{\stackrel{1}{\mathcal{T}}(\lambda), \stackrel{2}{\mathcal{T}}(\mu)\} & =[r(\lambda-\mu), \stackrel{1}{\mathcal{T}}(\lambda) \stackrel{2}{\mathcal{T}}(\mu)] \\
& +\stackrel{1}{\mathcal{T}}(\lambda) r(\lambda+\mu) \stackrel{2}{\mathcal{T}}(\mu)-\stackrel{2}{\mathcal{T}}(\mu) r(\lambda+\mu) \stackrel{1}{\mathcal{T}}(\lambda) .
\end{aligned}
$$

In the simplest case of the $4 \times 4$ rational $r$-matrix (3.2) matrix $\mathcal{T}(\lambda)$ depends polynomially on the parameter $\lambda$

$$
\mathcal{T}(\lambda)=\left(\begin{array}{cc}
\mathcal{A}(\lambda) & \mathcal{B}(\lambda) \\
\mathcal{C}(\lambda) & \mathcal{A}(-\lambda)
\end{array}\right), \quad \operatorname{deg} \mathcal{T}(\lambda)=\left(\begin{array}{cc}
2 n+1 & 2 n+1 \\
2 n-1 & 2 n+1
\end{array}\right) .
$$

Coefficients of the entries

$$
\begin{aligned}
& \mathcal{A}(\lambda)=\alpha \lambda^{2 n+1}+\mathcal{A}_{2 n} \lambda^{2 n}+\mathcal{A}_{2 n-1} \lambda^{2 n-1} \ldots+\mathcal{A}_{0}, \\
& \mathcal{B}(\lambda)=\lambda^{2 n+1}+\mathcal{B}_{n} \lambda^{2 n-1}+\mathcal{B}_{n-1} \lambda^{2 n-3} \ldots+\mathcal{B}_{1} \lambda, \\
& \mathcal{C}(\lambda)=\mathcal{C}_{n} \lambda^{2 n-1}+\ldots+\mathcal{C}_{2} \lambda^{3}+\mathcal{C}_{1} \lambda,
\end{aligned}
$$


are generators of the quadratic Poisson algebra (3.1). The leading coefficient $\alpha$ and $2 n+1$ coefficients of the $\operatorname{det} T(\lambda)$

$$
d(\lambda)=\operatorname{det} \mathcal{T}(\lambda)=Q_{2 n} \lambda^{4 n}+Q_{2 n-1} \lambda^{4 n-2}+\cdots+Q_{0} .
$$

are Casimirs of the bracket (3.1). Therefore, we have a $4 n+1$-dimensional space of the coefficients

$$
\mathcal{A}_{0}, \ldots, \mathcal{A}_{2 n}, \mathcal{B}_{1}, \ldots, \mathcal{B}_{n}, \mathcal{C}_{1}, \ldots, \mathcal{C}_{n}
$$

with $2 n+1$ Casimir operators $Q_{i}$, leaving us with $n$ degrees of freedom.

\subsection{Open lattices}

For so-called open lattices integrals of motion are given by the coefficients of the entry $\mathcal{B}(\lambda)$ :

$$
\mathcal{B}(\lambda)=\lambda^{2 n+1}+H_{1} \lambda^{2 n-1}+H_{2} \lambda^{2 n-3} \ldots+H_{n} \lambda, \quad\left\{H_{k}, H_{m}\right\}=0 .
$$

In the special action-angle representation [20, one has $n$ pairs of the action-angle variables:

$$
\mathcal{B}\left( \pm I_{m}\right)=0, \quad \omega_{m}=\eta^{-1} \ln \mathcal{A}\left(I_{m}\right), \quad m=1, \ldots, n,
$$

having the standard Poisson brackets (1.1).

As above the action variables $I_{k}$ are zeroes of the polynomial

$$
\mathcal{B}(\lambda)=\lambda^{2 n+1}+H_{1} \lambda^{2 n-1}+H_{2} \lambda^{2 n-3} \ldots+H_{n} \lambda=\lambda \prod_{m=1}^{n}\left(\lambda^{2}-I_{m}^{2}\right),
$$

integrals of motion $H_{m}$ are elementary symmetric function on $I_{m}^{2}$

$$
H_{1}=-\sum_{m=1}^{n} I_{m}^{2}, \quad H_{2}=\sum_{k \neq m}^{n} I_{k}^{2} I_{m}^{2}, \quad \ldots, \quad H_{n}=(-1)^{n} \prod_{m=1}^{n} I_{m}^{2}
$$

and the matrix $\mathbf{J}(1.2)$ is equal to

$$
\mathbf{J}=2\left(\begin{array}{ccc}
-I_{1} & \cdots & -I_{n} \\
I_{1} \sum_{m \neq 1} I_{m}^{2} & \cdots & I_{n} \sum_{m \neq n} I_{m}^{2} \\
\vdots & \ddots & \vdots \\
(-1)^{n} I_{1} \prod_{m \neq 1} I_{m}^{2} & \cdots & (-1)^{n} I_{1} \prod_{m \neq n} I_{m}^{2}
\end{array}\right) .
$$

As above all the functions $\phi_{j}(1.3)$ are functionally independent and one gets that open lattices related with the reflection equation algebra are maximally superintegrable systems.

We have to underline that functions $\omega_{k}$ (4.7) and, therefore, integrals of motion $\phi_{k}$ may be found without integration, i.e. using pure algebraic constructions. As above any action variables $I_{i}$ have additional polynomial integrals of motion $\mathcal{A}\left(I_{j}\right)$

$$
\left\{I_{i}, w_{j}\right\}=\left\{I_{i}, \ln \mathcal{A}\left(I_{j}\right)\right\}=0, \quad \Rightarrow \quad\left\{I_{i}, \mathcal{A}\left(I_{j}\right)\right\}=0, \quad i \neq j,
$$

but for the integrals $H_{k}$ one gets some combinations of logarithms and $I_{j}$ only. 


\subsection{Periodic lattices}

The theory of periodic lattices is based on the following construction of commutative subalgebras [9, 12]. Let us introduce the boundary matrix

$$
\mathcal{K}(\lambda)=\left(\begin{array}{cc}
a(\lambda) & 0 \\
c(\lambda) & d(\lambda)
\end{array}\right)
$$

whose entries $a(\lambda), d(\lambda)$ are polynomials with numerical coefficients and entry $c(\lambda)$ is arbitrary polynomial on $\lambda$. If the polynomial

$$
\tau(\lambda)=\operatorname{tr} \mathcal{K}(\lambda) \mathcal{T}(\lambda)=a(\lambda) \mathcal{A}(\lambda)+c(\lambda) \mathcal{B}(\lambda)+d(\lambda) \mathcal{A}(-\lambda)
$$

has $n$ independent dynamical coefficients $H_{1}, \ldots, H_{n}$ only, then

$$
\{\tau(\lambda), \tau(\mu)\}=0, \quad \Rightarrow \quad\left\{H_{i}, H_{j}\right\}=0, \quad i, j=1, \ldots, n .
$$

These Poisson involutive integrals of motion $H_{i}$ define the Liouville integrable systems or the periodic lattices related with the reflection equation algebra.

In the periodic case the $n$ pairs of the former action-angle variables (4.7) for the open lattices

$$
\mathcal{B}\left( \pm u_{m}\right)=0, \quad v_{m}=\mathcal{A}\left(u_{m}\right), \quad m=1, \ldots, n,
$$

are the simple separated variables, which satisfy to the following separated relations

$$
a\left(u_{j}\right) v_{j}+d\left(u_{j}\right) \operatorname{det} \mathcal{T}\left(u_{j}\right) v_{j}^{-1}=\tau\left(u_{j}\right) .
$$

The corresponding symplectic form in $(u, v)$-variables has the form (3.9). Therefore, we can apply the above consideration of periodic lattices related with the Sklyanin algebra to construction of the angle variables (3.10) in this case too.

As above the action variables $I_{m}$ coincide with integrals of motion $H_{m}$ and the matrix $\mathbf{J}$ (1.2) is identity matrix. Additional integrals of motion $\phi_{k}$ coincide with the angle variables $\omega_{m}$ (3.10), which are integrals of Abelian differentials on the hyperelliptic curve $\mu^{2}=P(\lambda)$ defined by (4.10), i.e. they are elliptic functions. So, all the periodic lattices related with the reflection equation algebra are formally maximally superintegrable systems, i.e. locally superintegrable only.

Recall, that reflection equation algebra (4.1) is related with a set of integrable systems that includes the generalized Toda lattices, the Kowalevski top and $X X X$ Heisenberg magnet with boundary conditions [20].

\section{Example 6 Open Toda lattice}

According to [9, 12] the $2 \times 2$ Lax matrix for the generalized open Toda lattice

$$
\mathcal{T}(\lambda)=\left(\prod_{k=1}^{n} L_{k}(\lambda)\right) \mathcal{K}_{-}(\lambda)\left(\prod_{k=1}^{n} L_{k}(-\lambda)\right)^{-1},
$$

where $L_{i}$ is given by (3.14) and

$$
\mathcal{K}_{-}(\lambda)=\left(\begin{array}{cc}
2 a_{2} \lambda^{2}-i a_{1} \lambda+a_{0} & \left(4 a_{2} \mathrm{e}^{q_{n}}-1\right) \lambda \\
0 & 2 a_{2} \lambda^{2}+i a_{1} \lambda+a_{0}
\end{array}\right)
$$

satisfies to the reflection equation algebra at $\alpha=0$ and $\eta=1$. Here $p_{i}, q_{i}$ are dynamical variables and $a_{k}$ are parameters.

All the open Toda lattices associated with classical root systems $\mathscr{B}_{n}, \mathscr{C}_{n}, \mathscr{B} \mathscr{C}_{n}$ and $\mathscr{D}_{n}$ are isomorphic to each other [18] and, therefore, we consider $\mathscr{B}_{n}$ root system only. In this case $a_{2}=a_{1}=0$ and at $n=2$ one gets

$$
\begin{aligned}
\mathcal{B} & =\lambda^{5}+H_{1} \lambda^{3}+H_{2} \lambda=\lambda\left(\lambda-I_{1}^{2}\right)\left(\lambda-I_{2}^{2}\right)= \\
& =\lambda^{5}-\left(p_{1}^{2}+p_{2}^{2}-2 \mathrm{e}^{q_{1}-q_{2}}-2 a_{0} \mathrm{e}^{q_{2}}\right) \lambda^{3}+\left(\left(p_{1} p_{2}-\mathrm{e}^{q_{1}-q_{2}}\right)^{2}-2 a_{0}\left(p_{1}^{2} \mathrm{e}^{q_{2}}+\mathrm{e}^{q_{1}}\right)\right) \lambda,
\end{aligned}
$$


such that

$$
H_{1}=-I_{1}^{2}-I_{2}^{2}, \quad H_{2}=I_{1}^{2} I_{2}^{2}, \quad \phi_{1}=-\frac{I_{1} \omega_{1}-I_{2} \omega_{2}}{2\left(I_{1}^{2}-I_{2}^{2}\right)}, \quad \phi_{2}=\frac{I_{1} \omega_{2}-I_{2} \omega_{1}}{2 I_{1} I_{2}\left(I_{1}^{2}-I_{2}^{2}\right)} .
$$

So, the Hamiltonian system with the hamiltonian $H_{1}$ has two independent integrals of motion $\mathrm{H}_{2}$ and

$$
\Phi_{2}=2 I_{1} I_{2}\left(I_{1}^{2}-I_{2}^{2}\right) \phi_{2}=I_{1} \omega_{2}-I_{2} \omega_{1}=-\left(I_{1} \ln \mathcal{A}\left(I_{2}\right)-I_{2} \ln \mathcal{A}\left(I_{2}\right)\right),
$$

where

$$
\mathcal{A}=-\mathrm{e}^{-q_{1}}\left(\lambda^{4}-p_{1} \lambda^{3}-\left(p_{2}^{2}+2 a_{0} \mathrm{e}^{q_{2}}+\mathrm{e}^{q_{1}-q_{2}}\right) \lambda^{2}+\left(p_{1} p_{2}^{2}-p_{2} \mathrm{e}^{q_{1}-q_{2}}+2 a_{0} p_{1} \mathrm{e}^{q_{2}}\right) \lambda\right)-a_{0} .
$$

Such as matrix $\mathbf{J}(1.2)$ has the special form, the additional integral of motion $\Phi_{2}$ has the form of the angular momentum in $(I, \omega)$-variables (see [17] and [3]). However at $n>2$ we will have completely another picture.

\section{Conclusion}

We discuss some constructions of the single valued integrals of motion on the whole phase space by using multi-valued action-angle variables.

For the Stäckel systems we use addition theorem for the construction of the polynomial additional integrals of motion starting with zero-genus hyperelliptic curves. The construction of the polynomial additional integrals of motion is an algebraic procedure for the open lattices related with the Sklyanin algebra or with the reflection equation algebra. On the other hand for the periodic lattices one gets the sums of integrals of first kind Abelian differentials on the hyperelliptic curves, which is locally defined only.

It will be interesting to consider inverse problem and try to find algorithm of construction action-angle variables starting with known additional polynomial integrals of motion.

The research was partially supported by the RFBR grant 06-01-00140.

\section{References}

[1] M. Agrotis, P. A. Damianou, C. Sophocleous, The Toda lattice is super-integrable, Physica A, v.365, p.235-243, 2006.

[2] V.M. Buchstaber, V.Z. Enolskii, and D.V. Leykin. Kleinian functions, hyperelliptic Jacobians and applications, volume 10 of Reviews in Mathematics and Mathematical Physics, pages 1-125, Gordon and Breach, London, 1997.

[3] L. Degiovanni, A note on the superintegrability of the Toda lattice, Preprint: arXiv:nlin/0606072, 2006.

[4] B.A. Dubrovin. Theta functions and nonlinear equations, Russ. Math. Surveys., v.36, p.11, 1981.

[5] A. Henrici, T. Kappeler, Global action-angle variables for the periodic Toda lattice, Preprint: arXiv:0802.4032, 2008.

[6] Yu. A. Grigoryev and A.V. Tsiganov, Symbolic software for separation of variables in the Hamilton-Jacobi equation for the L-systems, Regular and Chaotic Dynamics,, v.10(4), p.413-422, 2005.

wWw. maplesoft.com/applications/app_center_view . aspx?AID=1686

[7] M. N. Nekhoroshev, Action-angle variables and their generalizations, Trans. Moscow Math. Soc. v.26, p.180-198, 1972. 
[8] E.K. Sklyanin, The quantum Toda chain, Lecture Notes in Phys., v.226, p.196-293, 1985.

[9] E.K. Sklyanin, Boundary conditions for integrable quantum systems, J. Phys. A: Math. Gen., v.21, p.2375, 1988.

[10] E.K. Sklyanin, Separation of variables - new trends, Progr. Theor. Phys. Suppl., v.118, p.35, 1995

[11] F.A. Smirnov, Structure of matrix elements in quantum Toda chain, J. Phys.A: Math. Gen. v.31, p.8953-8971, 1998.

[12] V.V. Sokolov and A.V. Tsiganov, Commutative Poisson subalgebras for Sklyanin brackets and deformations of some known integrable models, Teor. Math. Phys., v.133(3), p.485-500, 2002.

[13] P. Stäckel. Comptes Rendus, v.116 and v.121, p.485, p.1284 and p.489, 1893 and 1895.

[14] P. Tempesta, P. Winternitz et al (Editors), Superintegrability in Classical and Quantum Systems, CRM Proceedings and Lecture Notes, v. 37 (AMS, Providence, RI, 2004).

[15] A.V. Tsiganov, The Stäckel systems and algebraic curves, J. Math. Phys., v.40, p.279-298, 1999.

[16] A.V. Tsiganov, Duality between integrable Stäckel systems, J. Phys.A: Math. Gen., v.32, p.7965-7982, 1999.

[17] A.V. Tsiganov, On the Drach superintegrable systems, J. Phys.A: Math. Gen., v.33, p.7407$7423,2000$.

[18] A.V. Tsiganov, Toda chains in the Jacobi method, Teor. Math. Phys., v.139(1), p.636-652, 2004.

[19] A.V. Tsiganov, A family of the Poisson brackets compatible with the Sklyanin bracket, J. Phys.A: Math. Theor. v.40, p.4803-4816, 2007.

[20] A.V. Tsiganov, The Poisson bracket compatible with the classical reflection equation algebra, accepted to Reg. Chaot. Dynamics, 2007, Preprint: arXiv:0709.0242, 